\title{
Estilos de gestión del conflicto interpersonal en las organizaciones
}

\author{
Lourdes Munduate, Juan Ganaza y Manuel Alcaide \\ Universidad de Sevilla
}

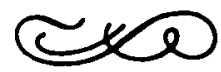

\begin{abstract}
Resumen
El estudio de los estilos de gestión del conflicto en las organizaciones, por parte de los directivos, es el objeto de análisis de este trabajo. Realizamos para ello la adaptación y validación del cuestionario ROCI-II (Rahim, 1983a), basado en una aproximación bidimensional -interés propio e interés por los demás - con cinco estilos distintos de gestión del conflicto interperonal -integración, servilismo, dominación, evitación y tendencia al compromiso-, y evaluamos las estrategias de gestión del conflicto empleadas por los directivos en la actualidad. Los resultados obtenidos confirman que la estructura de los cinco estilos distintos de gestión del conflicto se reproduce en nuestro contex to cultural, hallándose algunos datos de interés en relación a las variables demográficas consideradas en el presente estudio.
\end{abstract}

Palabras clave: conflicto interpersonal, estilos de dirección, organizaciones.

\section{Conflict management styles in organizations}

\section{Abstract}

The study intends to analyze conflict-management styles used by executives in organizations. The questionnaire ROCI-II (Rahim, 1983a) was adapted and validated for this purpose. This instrument is based on a two-dimensional approach -concern for one's own interest and concern for the interests of others - with five different interpersonal conflict-management styles - integration, obliging, dominating, avoiding and compromising-. We assess the conflict-management strategies used by executives at the present moment. The results obtained confirm that the five-style structure appears in our cultural context, and some interesting data are obtained with regard to demographic variables considered in the study.

Key words: Interpersonal conflict, styles of handling conflict, organization.

Dirección del autor: Dpto. de Psicología Social. Universidad de Sevilla. Apdo. 3128 - Sevilla.

Original recibido: Enero, 1991.

(C) 1993 by Aprendizaje, Revista de Psicología Social, 1993, 8 (1), 47-68 
Uno de los aspectos más destacados del conflicto en las organizaciones es que resulta consustancial a la propia vida y dinámica organizativa, pudiendo tener consecuencias tanto funcionales como disfuncionales para las distintas partes de la organización. A pesar de este potencial positivo, el conflicto es considerado a menudo como una amenaza a la eficiencia y efectividad organizacional (March y Simon, 1958), dada nuestra tendencia a considerar las organizaciones bajo el modelo del orden (Burrell y Morgan, 1979; Greenhalgh, 1987). No obstante, la creación de tensión y conflicto pueden ser estrategias organizacionales deseables, por lo que el conflicto y su resolución son eventos útiles y muy recurridos en el ámbito organizacional. Desde esta perspectiva, la problemática no reside por tanto en evitar o prevenir el conflicto, sino en dirigirlo del modo más efectivo posible para todas las partes implicadas.

El análisis de los estilos de gestión del conflicto por parte de los directivos, es el objeto de análisis de este trabajo. Revisamos el propio concepto de conflicto desde una perspectiva psicosocial, consideramos sus aspectos funcionales y disfuncionales, para centrarnos en la evaluación de las estrategias de gestión del mismo empleadas por los directivos en la actualidad.

\section{MARCO TEORICO Y CONCEPTUAL}

\subsection{La naturaleza del conflicto social}

Del modo más sencillo posible, Deutsch (1983: 47) observa que: «Un conflicto existe cuando se da cualquier tipo de actividad incompatible». De un modo más explícito, señala Smith (1987) que el conflicto social surge cuando las partes de una relación de interdependencia tienen aspiraciones incompatibles sobre algunos aspectos de sus acciones conjuntas. Pruitt (1981) lo define como un episodio en el que una parte trata de influir sobre el otro o sobre un elemento del entorno común, mientras la otra parte se resiste.

Estas «metas», «intereses» o «actividades incompatibles» que separan a las partes en la organización pueden variar considerablemente desde las diferencias objetivas basadas en los diferentes intereses económicos o roles estructurales y jerárquicos, que las partes ocupan y representan en las organizaciones, hasta una percepción de las diferencias mayormente subjetiva, impersonal o socialmente construida (Kochan y Verma, 1983). Efectivamente, y como constata Ibáñez (1988: 7-8), el aspecto más relevante de la definición sobre el conflicto es que «... la misma debe recurrir a la subjetividad de los actores sociales, y más concretamente a sus representaciones cognitivas, para diferenciarlo de otros procesos afines como la contraposición objetiva de intereses o las competiciones formalmente reguladas...».

Centrada la definición del conflicto - para las dificultades asociadas a la definición del conflicto, ver Marck y Snyder, 1957; Schmidt y Kochan, 1972; Bercovitch, 1972, y Alcaide, 1987- indicar que las partes implicadas pueden variar desde individuos a naciones. El conflicto puede surgir entre una pareja, entre hermanos, entre dos departamentos, en la comunidad de vecinos, entre la dirección y los empleados, entre naciones, etc. La diversidad entre las partes en conflicto es extensible al tamaño de la unidad - un departamento de cinco empleados o uno de 200 , por ejemplo-, y al número de partes implicadas - 
conflicto entre dos naciones o conflicto entre 10 vecinos-. En el ámbito de las organizaciones el conflicto puede ser clasificado como intrapersonal, interpersonal, intragrupal e intergrupal (Rahim, 1986).

El presente estudio se centra en el conflicto interpersonal entre dos partes - conflicto diádico-, y a pesar de que el conflicto intra- e intergrupos comparte algunas de las propiedades y dinámicas del conflicto interpersonal, también conlleva otras peculiaridades que no permiten reducirlo a este nivel. Efectivamente, tanto la intención de explicar la conducta intergrupal en base a principios derivados de la conducta interpersonal -ampliamente analizado y debatido por autores como Tajfel (1978), Brown y Turner (1981) y Stephenson (1984)como la generalización y la aplicación a contextos reales de los resultados de los experimentos de laboratorio con los que se han llevado a cabo dichas investigaciones no se han hallado exentos de críticas. Señalar, a su vez, que la discusión a nivel interpersonal no toma en consideración sus aplicaciones al nivel intrapersonal.

\subsection{La gestión del conflicto en las organizaciones}

Centrándonos por tanto en el ámbito de nuestro objetivo de análisis, se precisa indicar que el conflicto resulta prácticamente consustancial a la propia vida y dinámica organizativa, dándose a todos los niveles de la organización: se encuentra presente en las relaciones interpersonales, en las relaciones entre grupos, en las discusiones presupuestarias y en las luchas por la autonomía y el control. Es un proceso que emana de la rutina diaria, de las presiones estructurales y de los participantes en el entorno organizacional.

Como ha sido puesto de manifiesto por muy diversos autores (Pondy, 1967; Thomas, 1976; Pfeffer, 1981; Brown, 1983; Filley, 1975; Alcaide, 1987; Rahim, 1985,1990 ), el conflicto es un fenómeno que puede generar tanto consecuencias positivas y funcionales como provocar importantes perjuicios a los individuos, los grupos y/o las organizaciones. El progreso se logra a menudo mediante la implicación individual en una causa, y la creación de tensión y conflicto pueden ser estrategias organizacionales deseables. Como observa Raiffa (1982), algunas de las mayores mejoras sociales han sido el resultado de conflictos resueltos mediante fuerzas destructivas. Los deportes competitivos, los juegos de salón y los juegos de cartas son conflictos diseñados para añadir alicientes y estimular la vida cotidiana. La competición para el desarrollo en el mundo empresarial y la competición entre las compañías generan incentivos que ayudan al sistema a trabajar más eficientemente.

Merece mención en este contexto la referencia de Erika Apfelbaum (1990) a la concepción sobre el conflicto de autores como Park (1913/1950) y Lewin (1939/1948), en cuyos trabajos se denotan las implicaciones positivas y constructivas que se pueden derivar del mismo. Efectivamente, los artículos recogidos en el volumen póstumo de Lewin (1948) y paradójicamente titulado Resolving Social Conflicts propugnan precisamente una estrategia radicalmente distinta a la de la resolución de conflictos, cual es la implicación activa en la lucha por parte de los grupos minoritarios. Al referirse el artículo a las relaciones entre grupos minoritarios en términos de conflicto, Lewin propone que los mismos se impliquen activamente en una lucha que les lleve a afirmar y a recibir reconocimiento de su propia identidad. Observa Erika Apfelbaum, al respecto de esta propuesta de Lewin, que esto es cierto especialmente en aquellos grupos 
cuya supervivencia esté en peligro, como sucedía a los judíos en el momento en que Lewin formulaba su análisis.

Subraya Apfelbaum que el análisis realizado por Lewin en relación a las funciones positivas del conflicto es tan completo, incisivo y penetrante que parece sorprendente que nadie lo haya continuado o extendido, teórica o experimentalmente. Cita Apfelbaum a continuación el comentario de Coser (1956 cfr. Apfelbaum 1989: 268) a este respecto: «El temprano énfasis de Lewin en las funciones positivas del conflicto no ha tenido continuación en sus discípulos, que, sin embargo, han llevado mucho más lejos que él su énfasis en los aspectos disfuncionales de la conducta de conflicto».

Ciertamente, el conflicto es un aspecto ambiguo de la realidad social, y del mismo modo que puede resultar estimulante para crear nuevas ideas o nuevos medios de convivencia, a esta versión liberadora e iluminadora se añade la otra cara de la moneda, en la que priman las consecuencias dañinas o destructivas, por la que pueden peligrar amistades, familias, instituciones sociales que nos nutren e incluso la propia especie humana en la era actual. La comprensión y el control del conflicto social es, por consiguiente, una meta natural de la aplicación del conocimiento psicosocial (Smith, 1987).

No se trata por tanto de oponerse al conflicto en sí, ni considerarlo funcional o disfuncional en sí mismo, sino de gestionarlo del mejor modo posible. El principio de que «la dirección efectiva del conflicto resulta crítica para el éxito organizacional» es quizá la premisa mejor articulada de la mayoría de las teorías de la dirección (Putman, 1985).

Analizamos a continuación las principales propuestas sobre los diversos estilos empleados por los directivos en la gestión del conflicto en las organizaciones, con el objetivo de contrastar posteriormente el empleo de los mismos en nuestro contexto cultural.

\subsubsection{Los estilos de gestión del conflicto}

Aun cuando la relación entre cantidad de conflicto y otras variables organizacionales no ha sido establecida de un modo empírico y contrastado, autores como Brown (1983) proponen que dicha relación tiene la forma de una U invertida tal y como muestra en la Figura I. Un nivel o cantidad de conflicto excesivamente elevado provoca la presencia de resultados negativos, al mismo tiempo que un nivel excesivamente bajo suele generar una actitud de estancamiento que hace que los resultados alcanzados no sean tampoco satisfactorios.

Por dirección efectiva del conflicto no nos referimos sólo a la cantidad del mismo, sino también a las distintas modalidades de conducta conflictiva que pueden desplegar los participantes en una situación o episodio de conflicto.

Se han establecido, en este sentido, diversas tipologías relativas a los estilos de dirección del conflicto interpersonal. Podemos señalar, siguiendo a Rahim (1990), que la distinción y clasificación entre estilos se ha realizado a través de las siguientes aproximaciones:

1. La aproximación de dimensión única. Esta aproximación distingue entre dos modos o estilos de conducta conflictiva: la cooperación y la competición (Tjosvold, 1989).

2. La aproximación de los tres estilos. Una aproximación que distingue tres modos de manejar el conflicto: la no confrontación, la orientación hacia la solución y el control. 


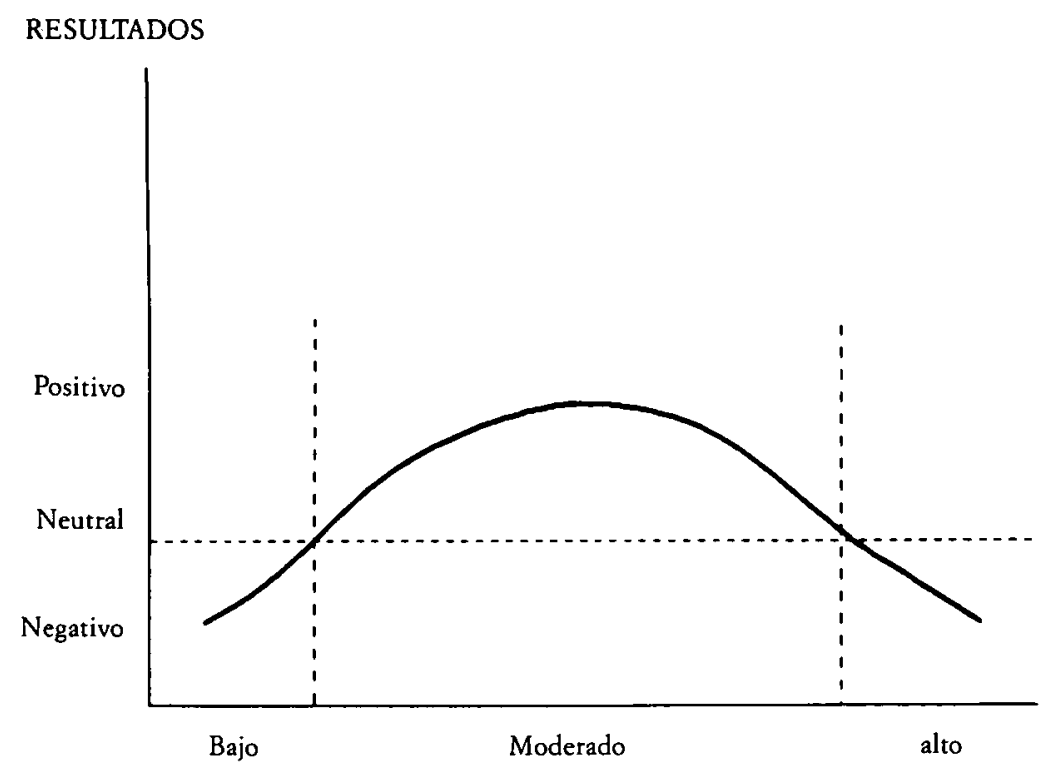

CANTIDAD DE CONFLICTO

3. La aproximación bidimensional con cuatro estilos. Una aproximación en la que se considera que la conducta conflictiva no puede verse como una conducta de una sola dimensión, sino que hay que tomar en consideración dos dimensiones distintas -el interés por los propios resultados y el interés por los resultados del otro-. De este modo, cualquier estilo de dirección del conflicto desarrollado por los sujetos será una combinación de estas dos dimensiones o componentes, que apuntan de bajo a alto. Los estilos presentados en esta aproximación (Pruitt, 1983) son cuatro: solución de problemas (alto interés por los resultados propios y alto interés por los resultados del otro), rivalizar (alto interés por los resultados propios y bajo interés por los resultados del otro), compla. cer (bajo interés por los resultados propios y alto interés por los resultados del otro) e inacción (bajo interés por los resultados propios y bajo interés por los resultados del otro).

4. La aproximación de dos dimensiones. Se trata también de una aproximación en la que se considera que la conducta conflictiva de una persona puede ser vista como una combinación de la inclinación presentada por dicha persona ante dos dimensiones diferentes. El origen de esta aproximación se encuentra en la catalogación realizada por Blake y Mouton (1964) sobre el interés que manifiestan los individuos sobre la producción o sobre las personas. Para estos autores las puntuaciones que se pueden alcanzar en ambas dimensiones varían entre un mínimo de 1 y un máximo de 9 , de modo que en teoría podrían existir 81 estilos diferentes de afrontar el conflicto. Sin embargo, Blake y Mouton (1964) terminan reduciendo su aportación a los cinco estilos más relevantes: dominar o forzar (estilo 9.1, 9 en interés por la producción y 1 en interés por las personas), retirada (estilo 1.1), suavizante (estilo 1.9), compromiso (estilo 5.5) y solución de problemas (estilo 9.9) (Figura II). 
FIGURA II

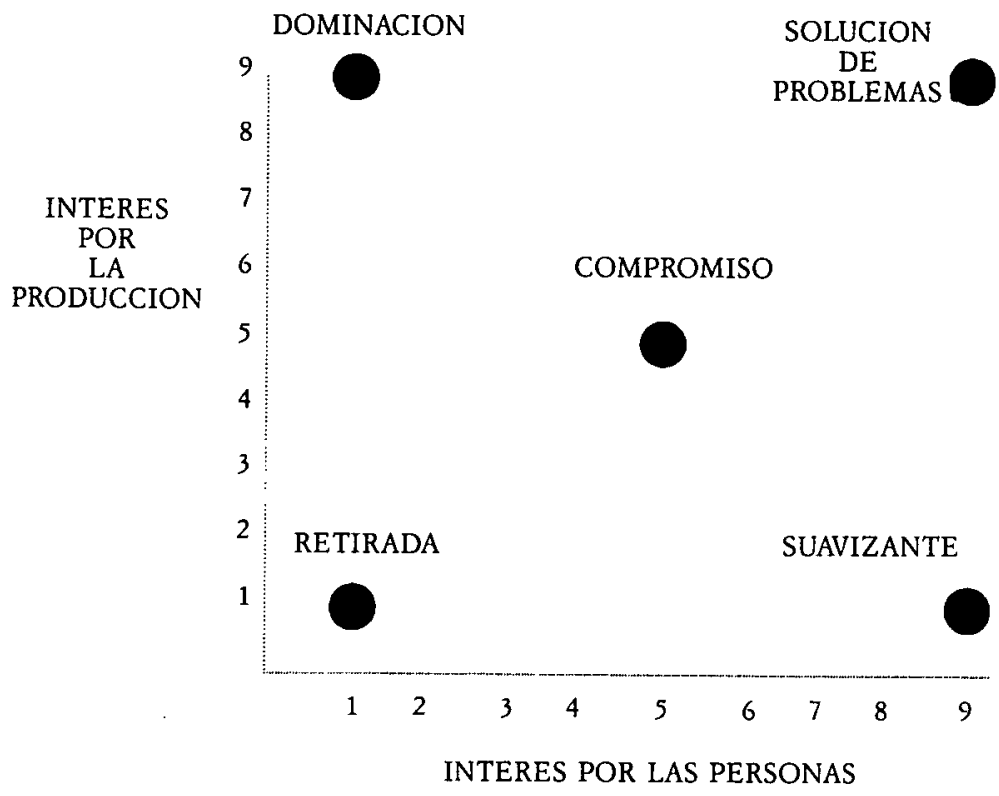

La conceptualización realizada por Thomas (1976) es una reinterpretación de la aproximación efectuada por Blake y Mouton (1964). Para Thomas (1976), las dos dimensiones básicas que determinan la distinción entre los distintos modos de manejo del conflicto son, por una parte, el grado de asertividad mostrado por el individuo, es decir, la medida en que intenta satisfacer sus propios intereșes; por otro lado, el grado de cooperatividad desplegado por el sujeto o el intento de satisfacer los intereses de la otra parte. En la conceptualización de Thomas, ambas dimensiones tienen dos puntuaciones extremas: asertivo vs. no asertivo y cooperador vs. no cooperador. La graduación efectuada por Blake y Mouton (1964) ya no está presente. Así pueden distinguirse también cinco estilos de conducta conflictiva: colaboración (asertivo y cooperador), competición (asertivo y no cooperador), evitación (no asertivo y no cooperador), acomodación (no asertivo y cooperador) y compromiso (medianamente asertivo y medianamente cooperador) (Figura III).

Existe una tercera reinterpretación de los estilos de manejo del conflicto similar a las realizadas por Blake y Mouton (1964) y Thomas (1976). Esta nueva clasificación es la realizada por Rahim y Bonoma (1979). Esta nueva conceptualización diferencia los estilos de dirección del conflicto en base a dos dimensiones, el interés propio y el interés de los otros. La primera dimensión explica la medida (alta o baja) en que un determinado sujeto trata de satisfacer su propio interés. En tanto que la segunda dimensión explica la medida (alta o baja) en que un individuo quiere o desea satisfacer el interés de los demás. Podría decirse que estas dos dimensiones ponen de manifiesto las orientaciones motivacionales de una determinada persona durante la situación de conflicto. Desde esta perspectiva, los cinco estilos posibles son: integración (alto interés propio y alto interés de los otros), servilismo (bajo interés propio y alto interés de los 
Figura III

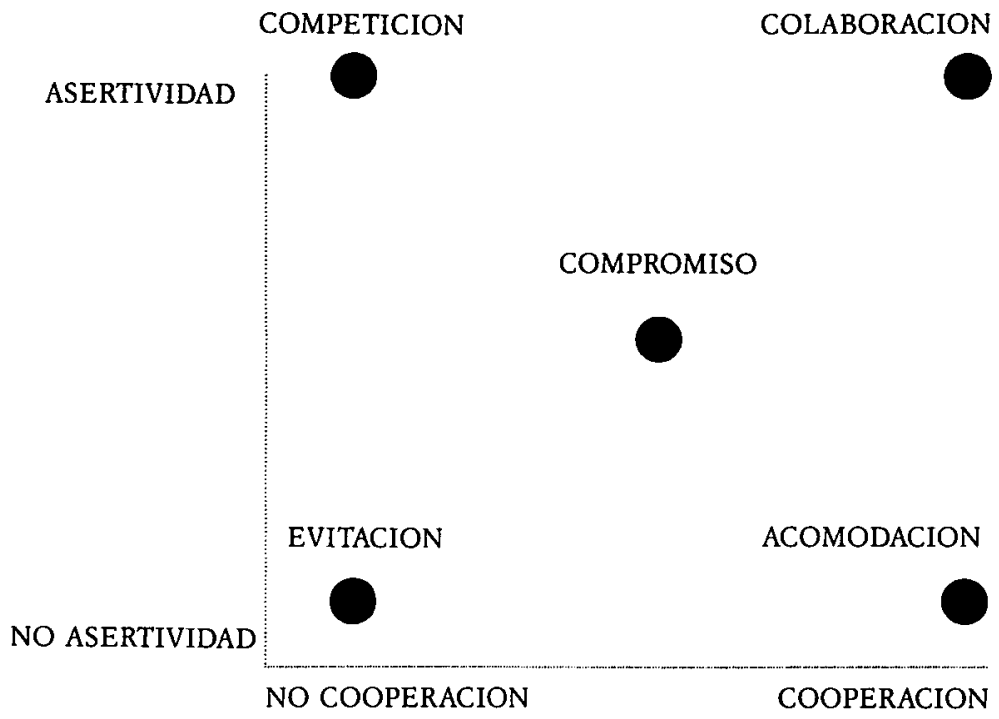

otros), dominación (alto interés propio y bajo interés de los otros), evitación (bajo interés propio y de los demás) y tendencia al compromiso (interés intermedio propio y por los demás) (Figura IV). Realizamos una breve descripción de estos estilos de gestión del conflicto interpersonal:

1. Integración: alto interés por uno mismo y los demás. Es un estilo que implica la colaboración entre los actores, por lo que puede llevar a la apertura, el intercambio de información y el examen de las diferencias existentes para tratar de llegar a una solución que sea aceptable para ambas partes.

Este estilo supone la comunicación directa entre las partes, lo que posibilita la resolución de problemas, conduciendo a soluciones creativas e imaginativas para los mismos.

2. Servilismo: bajo interés por uno y alto interés por los demás. Cuando una persona adopta este estilo, opta por no tomar en cuenta las diferencias existentes con la otra parte y se centra en enfatizar y destacar los aspectos comunes existentes entre ambos para procurar satisfacer el interés del otro actor. Supone en cierto grado un sacrificio para el protagonista que adopta este estilo.

3. Dominación: alto interés por uno y bajo interés por los demás. Es un estilo que ha sido identificado con una orientación de ganador-perdedor. Se trata de un tipo de persona que procurará por todos los medios lograr su objetivo y que, como consecuencia de ello, ignorará bastante a menudo las necesidades y expectativas de su oponente.

4. Evitación: bajo interés por los resultados propios y por los de los demás. Claramente asociada con la retirada y la esquivación del problema, a veces adopta la postura de posponer el problema hasta un momento más propicio. En otras ocasiones es la simple retirada de una situación que resulta amenazadora. 
FIGURA IV

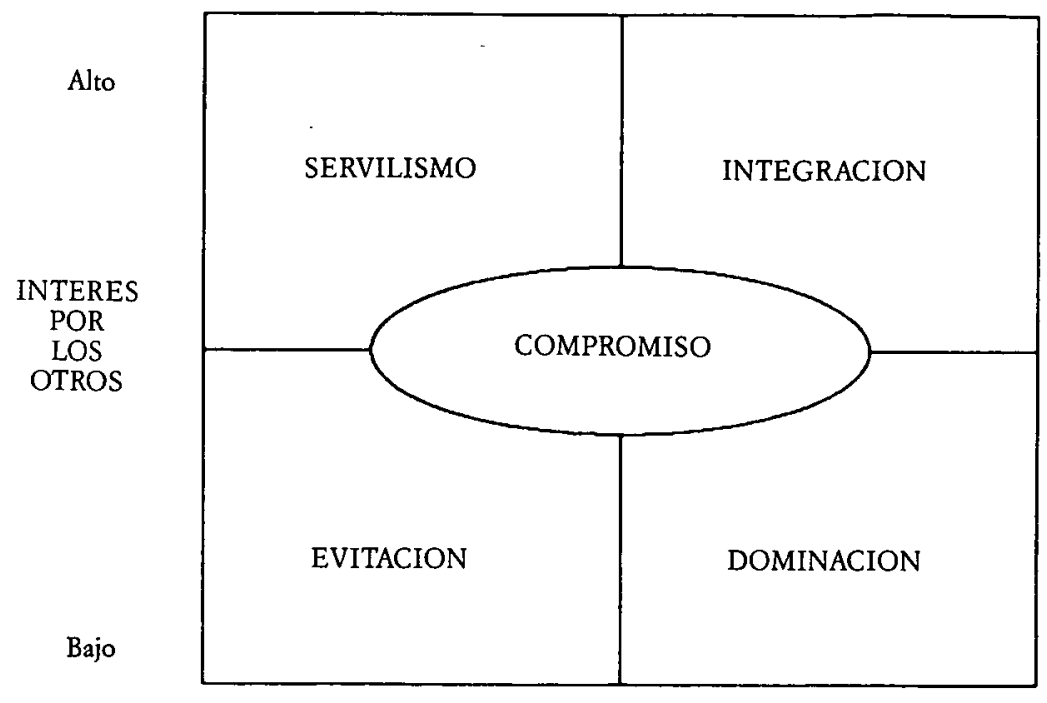

Bajo

Alto

INTERES PROPIO

5. Tendencia al compromiso: estilo intermedio en interés propio y de los demás. Aquí las partes ceden algo en su posición para poder tomar una decisión mutuamente aceptable para ambas. A veces significa intercambio de concesiones; otras, la búsqueda de una posición intermedia. En un reciente trabajo de Van de Vliert y Hordijk (1989) se identifica este estilo con la negociación distributiva, tal y como es definida por Walton y McKersie (1965) y por Bazerman y Lewicki (1983).

Durante bastantes años se han realizado diversas investigaciones (Ruble y Thomas, 1976; Cosier y Ruble, 1981; Ruble y Cosier, 1982; Rahim, 1983a, b; Chanin y Scheneer, 1984; Konovsky, Jaster y McDonald, 1989; King y Miles, 1990, Lee, 1990, y Van de Vliert y Kabanoff, 1990), que han tratado de poner de manifiesto la virtualidad de cada una de las cuatro aproximaciones anteriores. Dichas investigaciones han dado como resultado un apoyo importante a la aproximación bidimensional con cinco estilos.de dirección, ya sea en la versión de Thomas (1976), ya sea en la de Rahim y Bonoma (1979).

Esta situación ha propiciado el desarrollo de algunos instrumentos - descritos y analizados con extensión por Van de Vliert y Kabanoff (1990) y por King y Miles (1990) -, que basándose en la aproximación bidimensional de los cinco estilos, tratan de evaluar el grado en que se emplean estos diversos estilos de gestión del conflicto en la realidad organizacional. Entre estos instrumentos destacan el «Management of Differences Exercise» (MODE) de Thomas y Kilmann (1974), el «Organizational Conflict Communication Inventory» (OCCI) de Putman y Wilson (1982), y el «Rahim Organizational Conflict Inventory» (ROCCIII) de Rahim (1983a).

Se han realizado algunas contribuciones relevantes sobre los estilos de ges- 
tión del conflicto, centrándose básicamente en la incidencia de variables como el sexo, la formación, el estatus jerárquico, el área funcional y el rol desempeñado por la otra parte. Los hallazgos más relevantes sugieren que las mujeres tienden a ser más integradoras, evitadoras y con mayor tendencia al compromiso que los hombres, mostrándose al mismo tiempo menos servilistas que éstos (Rahim, 1983a; Kilmann y Thomas, 1977). A su vez, se observa que las mujeres presentan cierta tendencia a minimizar los disgustos emocionales y evitar tensiones, mostrando sus preferencias por los estilos menos competitivos de gestión del conflicto (Konovsky, Jaster y McDonald, 1989).

En cuanto a la educación, indicar que los sujetos tienden a ser más dominadores y menos evitadores a medida que tienen un mayor nivel educativo (Rahim, 1986), descendiendo a su vez el empleo del estilo integrativo, según desciende el nivel educativo de los mismos (Konovsky, Jaster y McDonald, 1989).

En relación al estatus jerárquico, se observa que los niveles inferiores son los que emplean con mayor asiduidad los estilos de servilismo y evitación (Rahim, 1986). En lo que concierne al área funcional, no se hallan diferencias significativas en base a esta variable, a excepción del estilo servicial, siendo los directivos de las áreas de personal e investigación y desarrollo los que más emplean este estilo, y los de dirección general los que menos recurren al mismo (Rahim, 1986).

Las propuestas realizadas sobre el rol desempeñado por la otra parte se centran en las relaciones con los superiores, los compañeros y los subordinados, argumentando en líneas generales que el estilo adoptado para la gestión de una determinada situación de conflicto varía en función del estatus relativo de las partes en confrontación (Rahim, 1983a; Lee, 1990). Se observa, concretamente, que los sujetos adoptan un estilo de dominación, esencialmente en la resolución de las diferencias con sus subordinados, pero escasamente con sus compañeros y superiores (Philips y Cheston, 1979). Se muestra, a su vez, una mayor tendencia al compromiso cuando ambas partes tienen una relación equitativa de poder ante una situación de conflicto (entre compañeros, por ejemplo) (Rahim, 1983a, 1986). Y finalmente, se da una mayor probabilidad de que los subordinados adopten un estilo de servilismo cuando la confrontación se da con un superior, más que cuando se da con un compañero o un subordinado (Rahim, 1986; Lee, 1990).

\section{OBJETIVOS E HIPOTESIS}

El objetivo general del presente estudio es el de constatar el grado en que se hallan presentes en la gestión del conflicto en nuestro contexto cultural los cinco estilos de dirección considerados como más útiles en la actualidad, para describir la conducta de los individuos en situaciones de conflicto.

Los objetivos concretos que nos planteamos son los siguientes: en primer lugar, la adaptación de un instrumento de medida de los estilos de gestión del conflicto a nuestro contexto cultural; en segundo lugar tratamos de constatar si la estructura de cinco estilos distintos en la gestión del conflicto se halla presente en los directivos de la muestra empleada. Tratamos también de verificar, finalmente, si se dan diferencias significativas entre los estilos de gestión más empleados en los directivos analizados en nuestra investigación y las halladas en otras investigaciones.

En función de la literatura previamente revisada, y de los resultados expuestos en el marco teórico de este trabajo, planteamos las siguientes hipótesis: 
$H$ 1: Se espera obtener una estructura empírica con cinco estilos distintos en la dirección del conflicto.

$H$ 2: Se espera que las mujeres obtengan puntuaciones significativamente superiores a los hombres en los estilos de integración, evitación y tendencia al compromiso, e inferiores en el estilo de servilismo.

H 3: Se darán diferencias significativas en los estilos de gestión del conflicto, según el nivel académico de los sujetos, en la siguiente dirección: a menor nivel académico, los sujetos se mostrarán menos integradores y evitadores y a su vez, a mayor nivel académico, los sujetos se mostrarán más dominadores.

H 4: Los directivos que ocupen niveles jerárquicos inferiores tenderán a ser significativamente más servilistas y evitadores que aquellos que ocupen posiciones más elevadas.

H 5: No se hallarán diferencias significativas en los estilos de gestión del conflicto, en base al área funcional en el que los sujetos lleven a cabo su actividad laboral.

H 6: Se darán diferencias significativas en los estilos de gestión del conflicto, según el rol jerárquico - jefe, subordinado, compañero- ocupado por la otra parte, en la siguiente dirección: los sujetos tenderán a ser significativamente más servilistas con sus jefes y a usar significativamente más la dominación con sus subordinados, mientras que tenderán al compromiso con sus compañeros.

\section{METODOLOGIA}

\subsection{Muestra}

La muestra está formada por 226 sujetos que desempeñan tareas de naturaleza directiva, con una media de 40 años de edad, y pertenecientes a diversas organizaciones que engloban los sectores de producción, sanidad y servicios, de la provincia de Sevilla.

\subsection{Variables e instrumentos}

Las variables demográficas consideradas en el presente estudio son: sexo, nivel de estudios, estatus jerárquico, área funcional y rol jerárquico del otro participante. La operacionalización y distribución de las mismas es la siguiente: 1) el sexo se distribuye en 196 hombres y 30 mujeres, 2) el nivel de estudios de los participantes se estructura en ocho categorías: estudios primarios $(\mathrm{N}=9)$ ), E.G.B. $(\mathrm{N}=13)$, F.P.1 $(\mathrm{N}=8)$, B.U.P. $(\mathrm{N}=17)$, F.P.2 $(\mathrm{N}=20)$, C.O.U. $(\mathrm{N}=22)$, Titulación media ( $(\mathrm{N}=69)$, Titulación superior $(\mathrm{N}=66) ; 3)$ el estatus jerárquico consta de representantes de los tres niveles organizacionales considerados: alto $(\mathrm{N}=47)$, medio $(\mathrm{N}=142)$ y bajo $(\mathrm{N}=36)$, con un tiempo medio en el desempeño de estos puestos directivos de 23, 20 y 13 años, respectivamente; 4) los sujetos pertenecen a cinco diferentes áreas funcionales: producción $(\mathrm{N}=29)$, marketing $(\mathrm{N}=16)$, contabilidad $(\mathrm{N}=35)$, dirección general $(\mathrm{N}=32)$ y otros $(\mathrm{N}=113)$; y 5) en el rol jerárquico del otro participante se incluyen, superiores $(\mathrm{N}=77)$, subordinados $(\mathrm{N}=73)$ y compañeros $(\mathrm{N}=76)$.

Para la consecución de los objetivos anteriormente planteados en relación a la evaluación de los estilos de gestión del conflicto empleados por los directivos de la muestra, y tras la revisión de los instrumentos actualmente relevantes 
- MODE, OCCI, ROCI-II-, optamos por adaptar a nuestro entorno cultural el instrumento ROCI-II (Rabim Organizational Conflict Inventory - II), debido fundamentalmente a los mayores niveles de fiabilidad y confianza mostrados por el mismo en los estudios realizados al respecto (Rahim, 1986; Knapp, Putman y Davis, 1988; King y Miles, 1990, y Van de Vliert y Kabanoff, 1990). En una reciente revisión sobre los estudios desarrollados utilizando el ROCIII, desde su aparición en 1983, Weider-Hatfield (1988) compara los coeficientes de la consistencia interna para cada una de las escalas, hallada en ocho estudios distintos, variando el alfa de Cronbach en el total de las escalas en los ocho estudios desde .50 a .95 , con una estimación alfa media de .79 , lo que muestra una relativa buena consistencia interna del cuestionario. A su vez, los análisis sobre los niveles de deseabilidad social mostrados por los sujetos en este cuestionario autoadministrado indican una gran insensibilidad al respecto (Rahim, 1983b; Weider-Hatfield, 1988).

\subsection{Adaptación del cuestionario}

El instrumento original fue diseñado en base al repetido feedback de los sujetos y al análisis factorial de los 35 ítems originales, 7 ítems para evaluar cada estilo de gestión del conflicto.

El ROCI-II consta de tres formas separadas - A, B y C-, que se diferencian sólo en sus referencias al conflicto con el jefe (forma $A$ ), con los subordinados (forma B) o con los compañeros (forma $\mathrm{C}$ ), respectivamente.

El análisis factorial de los 35 ítems de conflicto realizado por Rahim (1983a) extraía ocho factores, de los cuales los cinco primeros eran consistentes con las expectativas previas de Rahim y Bonoma (1979) sobre la aproximación bidimensional de los cinco estilos. Para el instrumento definitivo - y utilizado en el estudio que se presenta- se retuvieron los 28 ítems con pesos factoriales $\geqslant .40$.

Para responder a cada uno de los 28 ítems se utiliza una escala Likert de 5 puntos, donde los valores superiores representan una mayor utilización de un estilo conflictivo.

El ROCI-II también solicita información relativa a la edad, el sexo, el área funcional, el nivel organizacional y la educación.

El instrumento original (ROCI-II) fue traducido y adaptado al castellano, en sus tres formas $-\mathrm{A}, \mathrm{B}$ y $\mathrm{C}$ - Tras una primera traducción, se procedió a realizar una prueba de interpretación y comprensión entre varios ejecutivos. A partir de las indicaciones pertinentes de estas personas y de los errores y fallos de interpretación detectados en estas pruebas, se modificaron los ítems problemáticos, pasando finalmente el cuestionario a un experto o juez, con la finalidad de corroborar la asignación de cada ítem al estilo de gestión pertinente, como corresponde en la versión original. Posteriormente, los cuestionarios adaptados fueron distribuidos - proprocionalmente en las formas A, B y C-entre 310 ejecutivos, quedando la muestra definitiva constituida por 226 directivos.

\subsubsection{Resultado de la adaptación}

El ROCI-II, traducido y adaptado, obtuvo un coeficiente alfa de Cronbach para la escala total de .78. La fiabilidad estimada para las cinco escalas de los estilos de gestión en el instrumento adaptado, y comparado con la fiabilidad del instrumento original, se muestra en la Tabla 1. 
TABLA 1

Análisis de las escalas

\begin{tabular}{lccc}
\hline & $\begin{array}{c}\text { Fiabilidad del instrumento } \\
\text { original (ROCI-II) }\end{array}$ & $\begin{array}{c}\text { Fiabilidad del instrumento } \\
\text { adaptado (ROCI-II) }\end{array}$ \\
\hline $\begin{array}{c}\text { Escalas de los } \\
\text { estilos de gestión }\end{array}$ & $\begin{array}{c}\text { Cronbach } \\
\text { Alpha }\end{array}$ & $\begin{array}{c}\text { Spearman- } \\
\text { Brown }\end{array}$ & $\begin{array}{c}\text { Cronbach } \\
\text { Alpha }\end{array}$ \\
\hline Integración & .77 & .73 & .77 \\
Servilismo & .72 & .71 & .76 \\
Dominación & .72 & .71 & .75 \\
Evitación & .75 & .71 & .70 \\
Compromiso & .72 & .67 & .62 \\
\hline
\end{tabular}

Como podemos observar en esta tabla, la consistencia interna estimada del instrumento adaptado es satisfactoria, siendo la eścala de integración la que obtiene una mayor puntuación, con un alfa de .77, y el de compromiso el que obtiene una menor puntuación con un alfa de .62. La tendencia de estas dos escalas coincide con la mostrada por los análisis del instrumento original, donde la escala de integración obtiene un alfa de Cronbach de .77, una fiabilidad SpearmanBrown de .73 , y la escala de compromiso un alfa de Cronbach de .72 y una fiabilidad Spearman-Brown de .67 .

Considerando que la escala de compromiso es la que obtiene una menor puntuación también en otros estudios - como se observa en la revisión realizada por Weider-Hatfield, 1988, sobre la utilización del ROCI-II-, el resto presenta una estimación adecuada, a pesar de que se hallan debajo del mínimo estándar de .80 sugerido por Nunnally (1978), para la consistencia interna.

No obstante, la consistencia interna hallada resulta sumamente favorable comparada con la de otros instrumentos - el alfa de Cronbach para los instrumentos de Hall (1969) y Thomas y Kilmann (1974), varía entre .39 y .73 y .43 y .71, respectivamente- a excepción del OCCI de Putman y Wilson (1982), que varía entre .76 y .93 .

\subsubsection{Estructura factorial del instrumento}

Las respuestas de los 226 directivos a los 28 ítems de conflicto fueron factorizados, mediante el análisis de factores principales con varias iteracciones y rotación varimax. El análisis mostró siete factores con eigenvalues $\geqslant 1.00$. Estos siete factores explican el $57,2 \%$ de la varianza.

Según se desprende de la investigación de Rahim (1983a), y confirmada entre otros trabajos por Patrick (1987, cf. Weider-Hatfield, 1988), los 28 ítems del cuestionario empleado en nuestro estudio deberían ser agrupados en cinco factores, y alcanzar pesos factoriales $\geqslant$ a .40 . Los resultados obtenidos - ver la Tabla 2- muestran que sólo uno de los ítems no presenta un peso factorial significativo, en ninguno de los factores; además tres ítems presentan pesos factoriales significativos, en los factores sexto y séptimo.

El primer factor - Integración - aparece con siete ítems, quedando configurado del mismo modo que el de Rahim (1983a). El segundo factor Evitación - aparece con cinco ítems, perdiendo uno con relación al original. El tercer factor - Dominación - aparece con cinco ítems, quedando igual que 
TABLA 2

Matriz de la Estructura Factorial $(N=226)$

Factores

$\begin{array}{lllllllll}\text { N. } & \text { Items } & \text { I } & \text { II } & \text { III } & \text { IV } & \text { V } & \text { VI } & \text { VII }\end{array}$

01. Ante una dificultad de trabajo con , intento analizar la situación con él para encontrar una solución aceptable para ambos.

$\begin{array}{lllllll}.56 & -.11 & -.16 & -.03 & .25 & .16 & -.04\end{array}$

04. Ante una situación problemática con ___ intento integrar mis ideas y las suyas para alcanzar una decisión conjunta.

$\begin{array}{lllllll}.62 & .09 & .00 & .05 & .11 & .02 & -.15\end{array}$

05. Trato de analizar con __ las soluciones a los problemas que nos beneficien a ambos.

$\begin{array}{lllllll}.61 & .08 & .05 & .06 & .16 & .09 & .15\end{array}$

12. Ante una situación problemática con , la información que intercambio con él es siempre auténtica.

$$
\begin{array}{lllllll}
.51 & -.09 & -.04 & .18 & -.11 & -.15 & .10
\end{array}
$$

22. Ante un problema de trabajo con trato de plantear claramente nuestros intereses, para que el problema sea resuelto de la mejor forma posible.

$\begin{array}{rrrrrrr}.66 & .08 & .15 & .09 & -.04 & -.09 & .14 \\ .72 & .01 & .10 & .08 & .17 & .33 & -.07\end{array}$

23. Colaboro con __ para llegar a soluciones aceptables para ambos.

28. Ante un problema de trabajo con , intento analizar conjuntamente la situación para lograr una adecuada comprensión del mismo.

$\begin{array}{lllllll}.68 & .06 & -.17 & .06 & .09 & .13 & -.00\end{array}$

06. Cuando se trata de problemas de trabajo, generalmente evito discutir abiertamente con

$\begin{array}{lllllll}-.04 & .63 & -.02 & -.18 & .09 & .26 & .01\end{array}$

16. Intento no mostrar desacuerdo con

$\begin{array}{rrrrrrc}.00 & .65 & -.08 & .27 & .09 & .07 & -.03 \\ .23 & .77 & -.00 & .21 & .04 & -.02 & .19\end{array}$

17. Evito tropiezos con

26. Trato de no mostrar mi desacuerdo para evitar problemas.

$\begin{array}{lllllll}-.14 & .61 & .15 & -.01 & .02 & .10 & -.10\end{array}$

27. Intento evitar situaciones enojosas y desagradables con

$\begin{array}{llllllll}.18 & .77 & -.08 & -.02 & .03 & .04 & .10\end{array}$

08. Procuro utilizar todos los recursos a mi alcance para que mis ideas sean aceptadas.

$\begin{array}{lllllll}.00 & .12 & .71 & .04 & .24 & -.06 & .29\end{array}$

09. Uso de mi prestigio profesional y de la autoridad conferida para presionar a mi favor.

$\begin{array}{lllllll}-.23 & -.05 & .69 & .06 & .04 & .05 & -.05\end{array}$

18. Uso mis conocimientos y experiencia profesional para que las decisiones me favorezcan. 
Factores

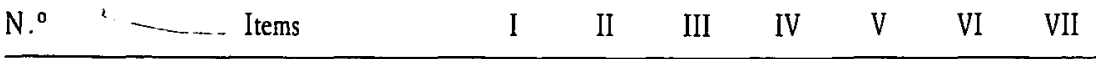

21. Ante una situación problemática con , generalmente me muestro

firme para procurar imponer mi punto de vista.

$\begin{array}{lllllll}.08 & -.09 & .67 & -.05 & -.04 & -.22 & .09\end{array}$

25. En ocasiones uso todos los recursos

a mi alcance, para ganar en una

situación de competencia

con

$\begin{array}{lllllll}-.06 & .02 & .71 & -.11 & -.03 & .09 & -.31\end{array}$

10. Generalmente actúo como

desea

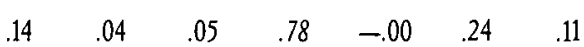

11. Generalmente acato los deseos

de

$\begin{array}{lllllll}.14 & -.03 & .04 & .83 & .04 & .10 & .09\end{array}$

13. Ante una dificultad de trabajo con , generalmente le hago concesiones.

$\begin{array}{lllllll}-.02 & .28 & .05 & .52 & .23 & -.04 & -.32\end{array}$

19. En el trabajo, generalmente acato las sugerencias de

$\begin{array}{llllllll}.28 & .12 & -.04 & .51 & .08 & .33 & -.27\end{array}$

07. Trato de encontrar caminos intermedios para avanzar hacia una solución.

$\begin{array}{lllllll}.20 & .16 & .06 & -.07 & .68 & .15 & -.06\end{array}$

14. Generalmente propongo un camino intermedio para romper con los puntos muertos.

$\begin{array}{lllllll}.09 & .05 & -.09 & .13 & .81 & .12 & .05\end{array}$

15. Ante problemas de trabajo procuro lograr acuerdos con _. a

$\begin{array}{lllllll}.38 & .05 & -.08 & .35 & .55 & -.14 & -.08\end{array}$

02. Ante un problema de trabajo, generalmente intento satisfacer las necesidades de

$\begin{array}{lllllll}.08 & .24 & -.11 & .26 & .09 & .69 & .12\end{array}$

24. En el trabajo, intento satisfacer las expectativas de

$\begin{array}{lllllll}.23 & .22 & .10 & .24 & .14 & .70 & -.08\end{array}$

03. En cuestiones de trabajo, trato de evitar que me pongan en un aprieto $\mathrm{e}$ intento no implicar a nadie en el problema que tengo con

$\begin{array}{lllllll}.09 & .20 & .06 & .07 & .04 & -.04 & .67\end{array}$

20. Al buscar soluciones a un problema de trabajo con , a veces tengo que ceder un poco para con. seguir algo.

\begin{tabular}{ccccccc}
.08 & .33 & .20 & .23 & .26 & -.29 & -.50 \\
\hline 5.23 & 2.89 & 2.34 & 1.75 & 1.51 & 1.21 & 1.08 \\
18.7 & 10.3 & 8.4 & 6.3 & 5.4 & 4.3 & 3.9 \\
18.7 & 29.0 & 37.4 & 43.6 & 49.1 & 53.4 & 57.2
\end{tabular}

Eigenvalue

Porcentaje de varianza

a La palabra jefe, subordinado o compañeros aparece en cada espacio en blanco en las formas $\mathrm{A}$, B y C, respectivamente. 
el original. El cuarto factor - Servilismo- contiene cuatro ítems, perdiendo dos con relación al original. El quinto factor - Compromiso- contiene tres ítems, perdiendo uno del original. El sexto factor contiene dos ítems que en el original pertenecen a Servilismo. El séptimo contiene un ítem que pertenece en el original a Evitación.

Como indicábamos anteriormente, Rahim (1983a), en su estudio original con 1.219 directivos, extrae ocho factores en un análisis factorial, eliminando siete ítems con pesos factoriales en los tres últimos factores. Los resultados obtenidos con el cuestionario adaptado ROCI-II apoyan la estructura de los cinco estilos, anulándose cuatro ítems del cuestionario original. Consideramos necesario, no obstante, realizar algunas sugerencias que derivan del análisis de los ítems que no cubren las expectativas previas. En este sentido, si atendemos conjuntamente los ítems anulados, tanto por parte de Rahim (1983a) como por nosotros, observamos algunos datos de interés. En relación al estilo de «Servilismo», si recogemos el ítem original anulado por Rahim (1983), que dice: $-« A$ veces, ayudo a mi ___ para tomar una decisión a su favor» (poniendo la palabra jefe, subordinado o compañero, en el espacio en blanco, según la modalidad del cuestionario)-, y los dos a eliminar en la presente adaptación -ítems número 2 y 24- observamos algunas similitudes entre sí, especialmente al compararlos con los otros cuatro ítems -ítems número $10,11,13$ × 19 - que se agrupan en el factor servilismo.

Así, mientras éstos se refieren a «acatar» y a «actuar» según los «deseos» y las «sugerencias» de la otra parte, y a realizar «concesiones con asiduidad», aquellos ítems hacen referencia a «concesiones» o «cesiones» para «satisfacer las necesidades» o las «expectativas» del otro, o para «ayudar» a éste. Es decir, en este caso se trataría de «ceder para ayudar o satisfacer a alguien» - a pesar de que no se obtenga nada a cambio, a diferencia del estilo de compromiso-, mientras que en los ítems agrupados en servilismo, se trataría de «ceder por los deseos de la otra parte», bien por costumbre o por desidia - si bien a diferencia del estilo de evitación, no se desprecia el interés para la otra parte-. Entendemos que esta situación puede estar indicando que en situaciones de bajo interés propio y elevado por los otros, los sujetos distinguen entre «el ceder por ceder, ante un mero deseo o sugerencia, aunque no esté presente la necesidad real en la otra parte», que sería un estilo complaciente - con tendencia a la evitación-, y «el ceder para satisfacer o ayudar a la otra parte, ante una determinada necesidad», que se aproximaría a un estilo considerado - tendente al compromiso donde el sujeto cede por implicación con la otra parte. El hecho de que no se agrupen bajo el factor de servilismo este tipo de ítems ayuda a delimitar la definición operacional de este estilo.

Proponemos a su vez, en relación al ítem número 20 , revisar la adaptación del mismo - concretamente el concepto de «give and take», entendido como «toma y dacai por los autores - ya que este ítem, controvertido desde su primera traducción, no parece tener un mismo significado en nuestro contexto cultural.

\section{RESULTADOS}

\subsection{Análisis diferenciales}

Para poner a prueba las hipótesis formuladas en base a las variables demográficas consideradas, hemos realizado análisis diferenciales, cuyos resultados 
se muestran en la Tabla 3. Observamos en la misma el tamaño de la muestra (N), la media (M) y la desviación típica (SD) de los cinco estilos de gestión del conflicto, clasificados por el rol jerárquico de la otra parte, el nivel organizacional, el área funcional, la educación y los resultados del análisis de varianza simple $(F)$.

En relación al rol jerárquico de los participantes, el análisis de varianza muestra que las diferencias significativas en el estilo de gestión del conflicto con el superior, los subordinados y los compañeros se producen en los estilos servilismo, dominación y compromiso. Los directivos de nuestra muestra tienden a usar un estilo servilista con mayor frecuencia con sus superiores que con sus subordinados y compañeros. Cuando la relación es más equitativa, tienden a mostrarse menos servilistas. Incluso emplean el compromiso en mayor proporción con sus compañeros que con sus jefes y más con éstos que con sus subordinados.

Aunque no se dan diferencias significativas en los estilos de gestión en base al nivel jerárquico, se pueden apuntar algunas tendencias. El estilo de evitación es más común entre los directivos de nivel intermedio que entre los de nivel inferior, y mayor entre éstos que entre los altos directivos.

El estilo integrador muestra diferencias significativas en relación al área funcional: los directivos que tienden más hacia la integración son los del departamento de marketing, y los menos integrativos los que pertenecen al de producción y al de dirección general. Los directivos del marketing son los que parecen emplear el estilo de evitación y el de compromiso con mayor frecuencia.

Se observan diferencias significativas en relación al nivel académico, en todos los estilos excepto en el de dominación. Los directivos que emplean más el estilo integrador son aquellos con un menor nivel de estudios, mientras que los más cualificados tienden a ser los menos integradores. Por otra parte, los que tienen una mayor formación son los que emplean con menor frecuencia el estilo de servilismo, evitación y compromiso. Se puede señalar como tendencia general que los directivos con mayor formación académica son los que emplean con menor frecuencia el estilo de evitación, del mismo modo que los de menor formación tienden a usar con mayor frecuencia el estilo de compromiso.

\subsection{Discriminación de grupos conocidos}

Otros estudios (Rahim, 1986) han indicado como variables discriminantes del estilo de gestión del conflicto interpersonal el rol jerárquico y el sexo. Con el objetivo de establecer la validez del instrumento ROCI-II para discriminar entre los diversos modos de gestión del conflicto en estos dos grupos conocidos -rol jerárquico de la otra parte y sexo-, se llevó a cabo un análisis discriminante en función de dichas variables.

Para llevar a cabo dicho análisis discriminante con relación a las distintas partes implicadas en la situación de conflicto - jefe, subordinados, compañeros-, optamos por realizar un análisis discriminante múltiple por pasos, con toda la muestra. El criterio usado para la selección de la escalación fue la distancia Mahalanobis.

Se obtuvieron dos funciones canónicas significativas que discriminaban entre los tres grupos. La primera función discriminante (Chi-cuadrado $=53.134$, $\mathrm{p} \leqslant .00001)$, tiene coeficientes discriminativos positivos para servilismo (1.09) y dominación (.32), y coeficientes negativos para integración (-.21) y evitación (-.51). El coeficiente de compromiso no es significativo. El grupo con centroide positivo es jefe (.63), y el grupo con centroide negativo es el de los subordi- 
Tabla 3

Valores muestrales de los estilos de gestión del conflicto $(N=226)$

\begin{tabular}{|c|c|c|c|c|c|c|c|c|c|c|c|c|c|c|c|c|}
\hline \multirow[b]{2}{*}{ Variables } & \multirow[b]{2}{*}{$\mathrm{N}$} & \multicolumn{2}{|c|}{ Integración } & \multirow[b]{2}{*}{$\mathrm{F}$} & \multicolumn{2}{|c|}{ Servilismo } & \multirow[b]{2}{*}{$\mathrm{F}$} & \multicolumn{2}{|c|}{ Dominación } & \multirow[b]{2}{*}{$\mathrm{F}$} & \multicolumn{2}{|c|}{ Evitación } & \multirow[b]{2}{*}{$\mathrm{F}$} & \multicolumn{2}{|c|}{ Compromiso } & \multirow[b]{2}{*}{ F } \\
\hline & & M & $S D$ & & M & $\mathrm{SD}$ & & M & SD & & M & SD & & M & SD & \\
\hline $\begin{array}{l}\text { Rol Jerárquico } \\
\text { Superior } \\
\text { Subordinado } \\
\text { Compañeros }\end{array}$ & $\begin{array}{l}77 \\
73 \\
76\end{array}$ & $\begin{array}{l}4.26 \\
4.16 \\
4.34\end{array}$ & $\begin{array}{l}.5060 \\
.5146 \\
.4336\end{array}$ & 2.46 & $\begin{array}{l}3.44 \\
2.92 \\
3.09\end{array}$ & $\begin{array}{l}.7151 \\
.5080 \\
.5238\end{array}$ & $15.10^{*}$ & $\begin{array}{l}3.09 \\
2.96 \\
2.76\end{array}$ & $\begin{array}{l}.6902 \\
.8722 \\
.7546\end{array}$ & $3.47^{\star}$ & $\begin{array}{l}3.30 \\
3.37 \\
3.46\end{array}$ & $\begin{array}{l}.7169 \\
.6989 \\
.7092\end{array}$ & .96 & $\begin{array}{l}3.78 \\
3.62 \\
3.88\end{array}$ & $\begin{array}{l}.5722 \\
.6167 \\
.5138\end{array}$ & $3.69^{\star}$ \\
\hline $\begin{array}{l}\text { Nivel Org. } \\
\text { Alto } \\
\text { Medio } \\
\text { Bajo }\end{array}$ & $\begin{array}{r}47 \\
142 \\
36\end{array}$ & $\begin{array}{l}4.25 \\
4.26 \\
4.28\end{array}$ & $\begin{array}{l}.5658 \\
.4818 \\
.4219\end{array}$ & .069 & $\begin{array}{l}3.18 \\
3.14 \\
3.17\end{array}$ & $\begin{array}{l}.6549 \\
.6409 \\
.5583\end{array}$ & .06 & $\begin{array}{l}3.01 \\
2.88 \\
3.04\end{array}$ & $\begin{array}{l}.7310 \\
.8144 \\
.7289\end{array}$ & .87 & $\begin{array}{l}3.25 \\
3.44 \\
3.30\end{array}$ & $\begin{array}{l}.7725 \\
.6944 \\
.6745\end{array}$ & 1.51 & $\begin{array}{l}3.79 \\
3.76 \\
3.74\end{array}$ & $\begin{array}{l}.5750 \\
.5462 \\
.6971\end{array}$ & .099 \\
\hline $\begin{array}{l}\text { Area funcional } \\
\text { Producción } \\
\text { Marketing } \\
\text { Contabilidad } \\
\text { Dir. General } \\
\text { Otros }\end{array}$ & $\begin{array}{r}29 \\
16 \\
35 \\
32 \\
113\end{array}$ & $\begin{array}{l}4.16 \\
4.60 \\
4.23 \\
4.15 \\
4.28\end{array}$ & $\begin{array}{l}.4615 \\
.3122 \\
.4832 \\
.5218 \\
.4908\end{array}$ & $2.77^{\star}$ & $\begin{array}{l}3.14 \\
3.58 \\
3.06 \\
3.17 \\
3.12\end{array}$ & $\begin{array}{l}.6782 \\
.5621 \\
.6523 \\
.6448 \\
.5935\end{array}$ & 2.13 & $\begin{array}{l}3.04 \\
3.01 \\
2.74 \\
3.10 \\
2.91\end{array}$ & $\begin{array}{l}.8160 \\
.5633 \\
.7673 \\
.7365 \\
.8119\end{array}$ & 1.07 & $\begin{array}{l}3.67 \\
3.48 \\
3.33 \\
3.27 \\
3.33\end{array}$ & $\begin{array}{l}.7298 \\
.7612 \\
.7668 \\
.7474 \\
.6517\end{array}$ & 1.61 & $\begin{array}{l}3.82 \\
4.03 \\
3.61 \\
3.72 \\
3.77\end{array}$ & $\begin{array}{l}.5644 \\
.4582 \\
.5617 \\
.6488 \\
.5665\end{array}$ & 1.56 \\
\hline $\begin{array}{l}\text { N. Académico } \\
\text { Primarios } \\
\text { E.G.B. } \\
\text { F.P.1 } \\
\text { B.U.P. } \\
\text { F.P.2 } \\
\text { C.O.U. } \\
\text { Titulación Media } \\
\text { Titulación Superior }\end{array}$ & $\begin{array}{r}9 \\
13 \\
8 \\
17 \\
20 \\
22 \\
69 \\
66 \\
\end{array}$ & $\begin{array}{l}4.49 \\
4.33 \\
4.46 \\
4.39 \\
4.21 \\
4.14 \\
4.36 \\
4.09 \\
\end{array}$ & $\begin{array}{l}.4102 \\
.3898 \\
.2342 \\
.4056 \\
.4959 \\
.4892 \\
.4825 \\
.5101 \\
\end{array}$ & $2.56^{\star}$ & $\begin{array}{l}3.28 \\
3.17 \\
3.56 \\
3.45 \\
3.21 \\
3.26 \\
3.17 \\
2.94 \\
\end{array}$ & $\begin{array}{l}.3239 \\
.6504 \\
.6177 \\
.5907 \\
.6767 \\
.6488 \\
.6012 \\
.6076 \\
\end{array}$ & $2.35^{\star}$ & $\begin{array}{l}3.31 \\
2.94 \\
3.13 \\
3.16 \\
2.84 \\
2.80 \\
2.90 \\
2.91 \\
\end{array}$ & $\begin{array}{l}.5665 \\
.8249 \\
.9052 \\
.9536 \\
.7736 \\
.7385 \\
.7480 \\
.7612 \\
\end{array}$ & .72 & $\begin{array}{l}3.78 \\
3.79 \\
3.81 \\
3.37 \\
3.28 \\
3.24 \\
3.41 \\
3.20 \\
\end{array}$ & $\begin{array}{l}.7412 \\
.7487 \\
.5555 \\
.7129 \\
.6648 \\
.7398 \\
.6634 \\
.6743 \\
\end{array}$ & $2.34^{\star}$ & $\begin{array}{l}4.03 \\
3.96 \\
4.03 \\
3.95 \\
3.65 \\
3.65 \\
3.82 \\
3.60 \\
\end{array}$ & $\begin{array}{l}.4779 \\
.6419 \\
.5651 \\
.5573 \\
.7517 \\
.3818 \\
.5531 \\
.5365\end{array}$ & $2.13^{*}$ \\
\hline Muestra Total & 226 & 4.26 & .4912 & & 3.15 & .6303 & & 2.94 & .7863 & & 3.38 & .7116 & & 3.76 & .5778 & \\
\hline
\end{tabular}


nados (-.35) y compañeros (-.31). Los directivos se muestran por tanto más servilistas y dominadores con sus jefes y más integradores y evitadores con sus subordinados y compañeros.

La segunda función discriminante (Chi-cuadrado $=10.77, \mathrm{p} \leqslant .03$ ) tiene coeficientes positivos para integración (.34) y compromiso (.64) y coeficiente negativo para dominación (-.45). Los coeficientes de obligación y evitación no son significativos. El grupo con centroide positivo es el de los compañeros (.27), y el grupo con centroide negativo, el de los subordinados (-.27). Ello indica que los directivos se muestran integradores con sus compañeros en las dos funciones discriminantes. En menor medida, los miembros de la organización se muestran servilistas con sus jefes:

Se llevó a cabo un segundo análisis discriminante con las cinco escalas como variables independientes y el sexo como variable dependiente. Como contamos sólo con 30 mujeres en la muestra, se seleccionaron 30 hombres aleatoriamente del resto de la muestra, combinándolos con las mujeres para el análisis. El análisis mostró una función canónica no significativa (Chicuadrado $=4.62, \mathrm{p} \leq .09$ ), por lo que no podemos establecer diferencias en los estilos de gestión del conflicto en función del sexo de los directivos, aunque sí podemos observar algunas tendencias, al obtenerse una centralidad de .29 y -.29 para las mujeres y los hombres, respectivamente, y un coeficiente de discriminación de $.91 \mathrm{y}-.79$ para las escalas de compromiso y evitación, respectivamente, sin que el resto de las escalas discriminase entre los dos grupos. Estos resultados, si bien no son significativos, parecen indicar que las mujeres muestran una mayor inclinación a usar el estilo de compromiso y menos el de evitación que los hombres.

\section{DISCUSION}

Con la finalidad de contrastar la estructura de los cinco estilos de gestión del conflicto interpersonal en nuestro contexto cultural, y las diferencias en el empleo de estos estilos en relación a determinadas variables demográficas, hemos llevado a cabo la adaptación y validación del cuestionario ROCI-II (Rahim, 1983a), obteniendo una fiabilidad satisfactoria que se confirma para cada una de las cinco subescalas del cuestionario. Los resultados obtenidos con el cuestionario adaptado indican que la estructura de los cinco estilos de gestión del conflicto se reproduce en los directivos sevillanos, confirmando la tendencia de los hallazgos de Rahim (1983a, b) y Van de Vliert y Kabanoff (1990). Los resultados se alejan por el contrario de las propuestas realizadas por WeiderHatfield (1988) -basándose en el análisis factorial y las correlaciones entre las cinco subescalas-, de considerar sólo tres estilos como los realmente evaluados por el ROCI-II: dominación, integración -incluyendo los ítems de integración y compromiso- y evitación, combinando los ítems de evitación y servilismo.

Podemos observar, a su vez, que el análisis conjunto de los ítems que no obtienen pesos significativos en el factor servilismo, tanto en el estudio de Rahim (1983b) como en el nuestro, muestra una orientación de los mismos a la implicación del sujeto con las necesidades o expectativas de la otra parte. Si nos centramos, por el contrario, en los ítems que se agrupan bajo el factor servilismo, este estilo queda definido por un tipo de conducta que cede ante los deseos y sugerencias de la otra parte, sin que ello implique interés por satisfacer las necesidades o expectativas del otro, se trata simplemente de «acatar» sus suge- 
rencias, probablemente por costumbre. Detectamos en este sentido una tendencia a evitar la mínima implicación, y a alejarse de la posibilidad de compromiso y consecuentemente de confrontación. Entendemos que los sujetos que emplean este estilo, definido en la dirección indicada, preferirán adoptar el estilo de evitación, antes que el estilo de compromiso, para eludir cualquier tendencia a la confrontación. Proponemos en este sentido para futuras investigaciones agrupar a los sujetos en función de la similitud de sus respuestas o perfiles, para tratar de identificar los principales patrones de los estilos de gestión del conflicto interpersonal.

La delimitación operativa detectada del estilo de servilismo podría explicar los resultados obtenidos por Van de Vliert y Hordijk (1989) y Van der Vliert y Kabanoff (1990), en relación a las dificultades del ROCI-II para discriminar claramente entre el estilo de evitación y el de servilismo - al ir anulándose precisamente los ítems que tienden a un mayor distanciamiento conceptual entre ambos-. Apoyamos a su vez la propuesta de Van der Vliert y Kabanoff (1990: 207) de «incrementar la distancia entre estas dos estrategias de no confrontación, contrastando el carácter reservado y no cooperativo de la evitación y el carácter abierto y cooperativo del servilismo». Consideramos, no obstante, en este último caso que esta idea de cooperación no debe suponer para el sujeto una noción de implicación y ayuda en la satisfacción de necesidades o expectativas de la otra parte.

Los resultados hallados en relación a las variables demográficas consideradas no permiten confirmar las diferencias halladas en los estudios previos sobre la incidencia del sexo de los participantes (Rahim, 1983a; Chanin y Schneer, 1984; Konovsky, Jaster y McDonald, 1989), coincidiendo con Renwick (1977), que tampoco halló diferencias en función de esta variable. Resulta preciso resaltar, a este respecto, que incluso entre los autores que encuentran diferencias significativas entre hombres y mujeres en el empleo de los diversos estilos de gestión del conflicto, no se da una coincidencia en la tendencia general de sus hallazgos. Así, mientras Rahim (1983a) encuentra que las mujeres emplean menos el estilo servilista, los resultados de Konovsky, Jaster y McDonald (1989) indican no obstante la preferencia de éstas por los estilos menos competitivos como el evitador y el servilista; Rahim (1983b) y Cardoso (1988) encuentran a su vez que las mujeres emplean más el estilo integrador, mientras Chanin y Schneer (1984) observan precisamente lo contrario. Para futuras investigaciones y ante este estado de discrepancias, nuestros resultados parecen apoyar las aportaciones de Cardoso (1988) que, ante esta problemática, aplica en su estudio - sobre los estilos de gestión del conflicto, también realizados en base a la aproximación bidimensional de cinco estilos- escalas de masculinidad-feminidad (De Díaz-Loving, et. al., cf. Cardoso, 1988), no hallándose relación entre el punto de vista dualístico de los atributos de personalidad masculinidad-feminidad, y un determinado sexo biológico.

Si bien se dan diferencias significativas entre el nivel académico y todas las escalas de gestión de conflicto, excepto en la de dominación, la hipótesis relativa a la relación entre nivel académico y estilo integrador no se confirma en nuestros directivos, puesto que a menor nivel académico muestran una mayor tendencia al empleo del estilo integrador, lo que no permite confirmar las diferencias halladas por Rahim (1986) en esta dirección. Por otro lado, sus conclusiones relativas al nivel académico y empleo del estilo de evitación y dominación (Rahim, 1986) se confirman sólo parcialmente: al igual que Rahim (1986) halla- 
mos que cuanto mayor es el nivel académico, menos se usa el estilo de evitación, y sin embargo, en el caso de nuestros directivos, no se dan diferencias significativas en relación al estilo de dominación. Otra característica relevante de los resultados de este estudio es la tendencia de los directivos de menor nivel académico a usar el estilo de compromiso.

El haber hallado diferencias significativas en relación al nivel académico, en todas las escalas excepto en la de dominación, mientras Rahim (1986) sólo encuentra en dominación y evitación, puede ser debido a que Rahim sólo considera cuatro niveles académicos y un quinto como «otros», mientras nosotros consideramos más niveles, y además estos niveles son inferiores a los considerados por Rahim - que considera a partir de licenciados «Bachiller»-, y también pueden ser debidas a las diferencias transculturales en la formación académica de los directivos americanos y sevillanos. De cualquier modo, los resultados relativos a la incidencia de la educación en el estilo de gestión adoptado indican que, dadas las diferencias halladas, se debería incluir, en futuras investigaciones sobre el tema, esta variable junto al rol jerárquico del otro participante, como dis criminante del estilo de gestión del conflicto interpersonal.

En relación al área funcional, se han hallado diferencias significativas en el estilo de integración, siendo los directivos del marketing los que más los emplean y los de producción y dirección general los que menos lo hacen. Las diferencias relativas al estilo de servilismo halladas por Rahim (1986) no han podido ser confirmadas, si bien se precisan reconsiderar las dimensiones de esta variable que no han conseguido aglutinar a los directivos de las áreas funcionales que operan en nuestro contexto cultural.

No se aprecian diferencias significativas en cuanto al nivel jerárquico ocupado por los directivos, demostrándose por el contrario diferencias en el estilo de gestión según el nivel jerárquico relativo de los mismos. Se dan efectivamente diferencias significativas en el empleo de los estilos de servilismo, compromiso y dominación, según el rol jerárquico desempeñado por la otra parte, confirmándose la hipótesis relativa al empleo del estilo servilista con los superiores (Rahim, 1986; Lee, 1990), y la relativa al empleo del estilo de compromiso con los compañeros (Rahim, 1983a, 1986). No se demuestra por el contrario que los directivos empleen más el estilo de dominación con los subordinados que con los compañeros y superiores, como habían propuesto Philips y Cheston (1979).

Tanto los trabajos anteriores sobre el tema como el nuestro han analizado independientemente la incidencia del poder relativo - rol jerárquico del otro participante- y el poder absoluto - status jerárquico - sobre la elección de un determinado estilo de gestión del conflicto por parte de los directivos. Los resultados hallados al respecto han desatado, no obstante, recientemente una polémica sobre la relevancia de una de estas variables sobre la otra. Así, mientras los resultados de Renwick (1975) indican que el status jerárquico no incide en la elección de uno de los cinco estilos de gestión y Mills y Chusmir (1988) proponen en esta misma línea que es el poder absoluto más que el relativo el que explica la elección de un determinado estilo por parte de los directivos, el trabajo de Lee (1990) demuestra que el poder relativo de las partes en conflicto determina la elección del estilo de gestión del mismo, mostrando a su vez los resultados de nuestro estudio esta misma orientación. Se precisan más investigaciones a este respecto, que permitan confirmar si se da una superioridad significativa del poder relativo sobre el absoluto, en la adopción de un determinado estilo de conducta ante una situación de conflicto. 


\section{Referencias}

Alcaide, M. (1987). Conflicto y poder en las organizaciones. Madrid, Centro de Publicaciones del Ministerio de Trabajo y Seguridad Social.

Apfrelbaum, E. (1990). Relaciones de dominación y movimientos de liberación. Un análisis del poder entre los grupos. En J. Morales, y C. Huici: Lecturas de psicologia social. Madrid: UNED.

Bazerman, M. H., y Lewicki, R. J. (Eds.) (1983). Negotiating in Organizations. Londres: Sage Publications.

BerCovitch,J. (1972). Conflict, peace and peace research. International Problems. Vol. 19, pp. 31-39.

Blake, R. R., y Mouton. J. S. (1964). The Managerial Grid. Houston, Gulf Publishing.

BROWN, L. D. (1983). Managing Conflict at Organizational Interfaces. Reading, Addison-Wesley.

Brown, R. J., y Turner, J. C. (1981). Interpersonal and intergroup behavior. En J. C. Turner, y H. Giles, (Eds.). Intergroup Behavior. Oxford: Blackwell.

BurRell, G., y Morgan, G. (1979). Sociological paradigms and organizational analysis. Londres: Heinemann.

Cardoso, R. (1980). Estilos en el manejo de conflictos en organizaciones mexicanas. Libro de simposios del I Congreso Iberoamericano y III Nacional de Psicología del Trabajo y de las Organizaciones. Madrid: Colegio Oficial de Psicólogos de Madrid.

Chanin, M. N., y Schneer, J. A. (1984). A study of the Relationship between Jungian Personality Dimensions and Conflict-Handling Behavior. Human Relations, 37, 863-879.

Cosier, R. A., y Ruble, T. L. (1981). Research on Conflict-Handling Behavior: An Experimental Approach. Academy of Management Joumal, 24, 816-831.

Deustch, M. (1983). Fifty years of conflict. En L. Festinger, (Ed.): Retrospections on social Psychology, New York: Oxford University Press.

Filley, A. C. (1975). Interpersonal Conflict Resolution. Glenview, Scott, Foresman and company.

Greenhalgh, L. (1987). Interpersonal conflicts in organizations. En C. L. Cooper y I. R. Robertson (Eds.): International review of industrial and organizational psychology, Nueva York: Wiley, pp. 229-271.

Hall, J. (1969). Conflict Management Survey: A Survey on one's Characteristics Reaction to and Hadling Conflict between Himself and others. Canoe, Telemetrics International.

IBÁN̄EZ, T. (1988). El conflicto social. Perspectivas clásicas y enfoque renovador. Boletín de Psicología, 18, pp. 7.20.

Kilmann, R. H., y Thomas, K. W. (1977). Developing a Forced-Choice Measure of ConflictHandling Behavior: The «MODE» Instrument. Educational and Psychological Measurement, 37, 309-325.

King, W. C., y MiLes, E. W. (1990). What we Know -and don't know- about measuring conflict: and examination of the ROCI-II and the OCCI conflicts instruments. Management Comunication Quarterly, Vol. 4, 2, pp. 222-243.

Knapp, M. L.; Putnam, L. L., y Davis, L. J. (1988). Measuring Interpersonal Conflict in Organizations: Where do we go from here? Management Comunication Quarterly, Vol 1, 3, pp. 414-429.

Kochan, T., y Verma, A. (1983). Negotiating in organizations Bleding industrial relations and organizational behavior approach. En M. H. Bazerman y R. Lewicki R. (Eds.): Negotiating in Organizations. Beverly Hills: Sage.

Konovsky, M. A.; Jaster, F., y McDonald, M. A. (1989). Using Parametric Statistics to Explore the Construct Validity of the Thomas-Kilmann Mode Survey. Management Communication Quarterly, 3, 268.290.

LEE, CH. W. (1990). Relative status of employees and styles of handling interpersonal conflict: An experimental study with Korean managers. The International Journal of Conflict Management, 1 (4), 327-340.

LEwIN, K. (1935). A dinamic theory of personality. Nueva York: McGraw-Hill.

LEwIN, K. (1948). Resalving social conflicts, cfr. Apfelbaum (1989). Op. cit.

March, J. G., y Simon, H. A. (1958). Organizations. Nueva York: Wiley.

MARCK, R. W., y SNYDER, R. C. (1957). The analysis of social conflict: toward an overview and synthesis. Joumal of Conflict Resolution, 1, pp. 212-248.

Mills, J., y Chusmir, L. A. (1988). Managerial conflict resolution styles: Work and home differences. Journal of Social Bebavior and Personality, 303-316.

Nunnally, J. M. (1978). Psychometric theory (2.a ed.). Nueva York, McGraw-Hill.

PARK, R. E. (1950): Race and Culture, cfr. E. Apfelbaum (1989). Op. cit.

Pffffer, J. (1981). Power in organizations. Marshfield: Pitman. 
Phillips, E., y Cheston, R. (1979). Conflict resolution: What works? California Management Review, 21 (4), 76-83.

Pondy, L. R. (1967). Organizational Conflict: Concepts and Models. Administrative Science Quarterly, 12, 296-320.

PruIT, D. G. (1981). Negotiation behavior. New York, Academic press.

Pruitr, D. G. (1983). Strategic Choice in Negotiation. American Bebavioral Scientist, 27, 167-194.

Putman, L. L. (1985). Bargaining as Organizational communication. En R. D. McPhee y P. $\mathrm{K}$. Tompkins (Eds.). Organizational comunication: Traditional themes and new directions. Beverly Hills: Sage.

PutMan, L. L., y WILSON (1982). Communicative strategies in organizational conflict: Reliability and validity of a measurement scale. En M. Burgoon (Eds.). Communication Yearbook, vol. 6, Beverly Hills: Sage, pp. 629-652.

Rahim, M. A. (1983a): Rabim organizational conflict inventory - II: Forms a, b, and c. Palo Alto, CA: Consulting Psychologists Press.

Raнim, M. A. (1983b): A Measure of Styles of Handling Interpersonal Conflict. Academy of Management Journal, 26, 368-376.

Rahim, M. A. (1985). A Strategy for Managing Conflict in Complex Organizations. Human Relations, 38, 81-89.

Rahim, M. A. (1986). Managing Conflict in Organizations. Nueva York, Praeger.

RaHim, M. A. (1990). A normative Theory of Conflict Management: Matching Styles of Handling Interpersonal Conflict with Situations. Debate on Alternative Theoretical Approacbes to Conflict Management. Third annual conference of the International Associa. tion for Conflict Management, Vancouver, Canadá.

Rahim, M. A., y Bonoma, T. V. (1979). Managing Organizational Conflict: A Model for Diagnosis and Intervention. Psychological Reports, 16, 143-155.

RAIFF, H. (1982). The art and Science of negotiation, Cambridge, Mass.: Harvard University Press. Wiley, pp. 229-271.

Renwick, P. A. (1975). Perception and Management of Superior-Subordinate Conflict. Organizational Behavior and Human Performance, 13, 444-456.

Renwick, P. A. (1977). The Effects of Sex Differences on the Perception and Management of Superior-Subordinate Conflict: An Exploratory Study. Organizational Behavior and Human Performance, 19, 403-415.

Ruble, T. L., y Cosier, R. A. (1982). A Laboratory Study of Five Conflict Handling Modes. En G. B. J. Bomers y R. B. Peterson (Eds.), Conflict Management and Industrial Relations. (pp. 158-171). Boston, Kluwer-Nihjoff.

Rubie, T. L., y Thomas, K. W. (1976). Support for a Two-Dimensional Model of Conflict Behavior. Organizational Bebavior and Human Performance, 16, 143-155.

Schmidt, S. M., y Kochan, T. A. (1972). Conflict: Towards conceptual clarity. Administrative Science Quarterly, Vol. 17, pp. 359-370.

S Mith, W. P. (1987). Conflict and negotiation. Trends and emerging issues. Joumal of applied social psychology. Vol 17, pp. 641-677.

STEPHENSON, G. M. (1984). Intergroup and interpersonal dimension of bargaining and negotiation. En H. Tajel (Ed.): The social dimension. Cambridge: Cambridge University Press.

TAJFEL, H. (1978). Differentiation between social groups: Studies in the social psychology of intergroup relations. Londres: Academic Press.

Thomas, K. W. (1976). Conflict and Conflict Management. En M. F. Dunnette (Ed.), Handbook of Industrial and Organizational Psychology, (pp. 889-935). Chicago, Rand McNally.

Thomas, K. W., y Kilmann, R. H. (1974). Thomas-Kilmann Conflict Mode Instrument. Nueva York, Xicom.

Tjosvold, D. (1989). Interdependence Approach to Conflict Management in Organizations. En M. A. Rahim (Ed.), Managing Conflict: An Interdisciplinary Approach (pp. 41-50). Nueva York, Praeger.

Vande Vliert, E., y Hordjk, J. W. (1989). A Theoretical Position of Compromising Among other styles of Conflict Management, Journal of Social Psychology, 129 (5), pp. 681-690.

Vande Vliert, E., y Kabanoff, B. (1990). Toward Theory-Based Measures of Conflict Management. Academy of Management Joumal, 33, 199-209.

W alton, R. E., y McKersie, R. B. (1965). A Behavioral Theory of Labor Negotiations. Nueva York, McGraw-Hill.

Weider.HATfieid, D. (1988). Assesing the Raghim Organizational Conflict Inventory-II (ROCI-II). Management Comunication Quarterly, Vol. 1, n. ${ }^{\circ}$ 3, pp. 350-366. 